\title{
"EL EFECTO SOBRE EL TIPO DE CAMBIO Y LAS TASAS DE INTERES DE LAS INTERVENCIONES EN EL MERCADO CAMBIARIO Y DEL PROCESO DE ESTERILIZACION".
}

\author{
Diciembre de 1997
}

\begin{abstract}
Alejandro Werner
(Agradezco los valiosos comentarios de Agustín Carstens Carstens, Moisés Schwartz Rosenthal y Pascual O’Dogherty Madrazo así como la valiosa colaboración de Jorge Pastrana Villegas. Por último quisiera agradecer a Gabriela Estevanez Cabral por su cooperación en la elaboración de este trabajo. Las opiniones contenidas en este documento corresponden exclusivamente al autor y no representan el punto de vista del Banco de México.)
\end{abstract}

Documento de Investigación No. 9706

\section{Dirección General de Investigación Económica}

Banco de México 


\section{RESUMEN}

Este trabajo estudia el impacto sobre el tipo de cambio y las tasas de interés tanto de las intervenciones del Banco de México realizadas en el mercado cambiario a través del mecanismo de opciones, así como de la política de esterilización que ha seguido el Instituto Central. De estos ejercicios se concluye que hasta el momento, no se ha detectado un efecto importante de las intervenciones en el mercado cambiario y de su esterilización subsecuente en la determinación del tipo de cambio y las tasas de interés. Sin embargo, los resultados de las estimaciones efectuadas indican que durante los últimos meses de 1997, el efecto de las operaciones de esterilización sobre las tasas de interés fue estadísticamente significativo pero de una magnitud muy pequeña. Finalmente, se encontró cierta evidencia que durante los períodos en los cuales existe la posibilidad de que ocurran estas intervenciones, las tasas de interés son ligeramente más elevadas que en los períodos en los que esta posibilidad no se presenta. Este resultado puede reflejar ciertas distorciones de caracter temporal que surgen en el mercado de dinero al realizarse las operaciones de esterilización. 
I.

II.

II.1 Efectos de las intervenciones en el mercado

Introdicción

Intervención en el mercado cambiario. de cambios a través del mecanismo de opciones.

III. Conclusiones.

IV. Bibliografía: 


\section{I.- Introducción:}

Este trabajo estudia el impacto sobre el tipo de cambio y las tasas de interés tanto de las intervenciones del Banco de México realizadas en el mercado cambiario a través del mecanismo de opciones, así como de la política de esterilización que ha seguido el Instituto Central.

Para entender el proceso de determinación del precio de una divisa se puede argumentar que éste es similar al de cualquier otro activo. Por lo tanto, este precio estará determinado por sus fundamentos y por las expectativas sobre la evolución futura de éstos que mantienen los agentes económicos. La forma en la cual los agentes generan dichas expectativas caracterizará en buena medida el comportamiento del precio del activo. Si suponemos que los agentes tienen expectativas racionales, el precio del activo cambiará cada vez que se reciba información adicional sobre el nivel actual y el comportamiento futuro de los fundamentos que determinan el precio del activo.

Las señales que los agentes económicos reciben sobre el nivel actual y el curso futuro de los fundamentos provienen, principalmente, de las acciones y señales que envían las autoridades monetarias y fiscales y de los anuncios que se hacen sobre los resultados recientes del comportamiento de las variables macroeconómicas.

Este trabajo se enfoca en el estudio de los efectos sobre el tipo de cambio y las tasas de interés de las intervenciones del Banco de México en el mercado cambiario y su esterilización subsecuente. Cuando las autoridades monetarias llevan a cabo una intervención en el mercado cambiario, se podrían alterar las expectativas de los agentes en cuanto al comportamiento futuro de los determinantes fundamentales del tipo de cambio y las tasas de interés, afectándose el comportamiento de éstas variables.

En el documento se describen, en primer lugar, los mecanismos a través de los cuales las intervenciones, esterilizadas y no esterilizadas, pueden afectar el nivel del tipo de cambio y de las tasas de interés. Como en el caso de México las intervenciones en el mercado cambiario siempre han sido esterilizadas, se profundiza en el estudio de este tipo de intervenciones. Finalmente, se realizó una evaluación econométrica del impacto sobre el tipo de cambio y las tasas de interés 
de estas intervenciones y se concluye que el efecto, hasta finales de 1997 cuando menos, ha sido nulo (o extremadamente pequeño). 


\section{II.- Intervención en el mercado cambiario.}

Existen dos tipos de intervenciones en el mercado cambiario: esterilizadas y no esterilizadas. La única diferencia entre ellas es que en la primera la autoridad monetaria elimina la expansión de liquidez que se origina por la intervención en el mercado cambiario. Por otro lado, las intervenciones no esterilizadas tienen un impacto sobre la oferta monetaria, debido a que no se cancela la expansión monetaria que genera la intervención.

La teoría monetaria tradicional, aplicada a la determinación del tipo de cambio, indica que las intervenciones no esterilizadas afectan el nivel del tipo de cambio de manera proporcional al cambio en las ofertas relativas de dinero, de igual manera que un cambio exógeno en estas ofertas. Por extensión lógica, la teoría monetaria tradicional indica que las intervenciones esterilizadas no deberían tener impacto sobre el tipo de cambio, pues por virtud de la esterilización no se estarían afectando las ofertas relativas de dinero.

Si la teoría monetaria tradicional no se sostiene, las intervenciones esterilizadas podrían afectar el comportamiento del tipo de cambio y de las tasas de interés. Estos efectos serían menos directos y más controvertidos que los que se presentan cuando se realiza una intervención no esterilizada. En la literatura se han mencionado dos mecanismos adicionales al monetario tradicional a través de los cuales una intervención esterilizada pudiera llegar a afectar al tipo de cambio: el efecto portafolio y el efecto señal. Estos se explican a continuación.

a) Efecto Portafolio: En los modelos de portafolio de determinación del tipo de cambio, se supone que los inversionistas diversifican su portafolio en base a los retornos esperados de las alternativas de inversión y a la varianza de éstos. De acuerdo a este enfoque, las intervenciones esterilizadas, al cambiar la composición de los activos en los portafolios, afectan el tipo de cambio (o a las tasas de interés) al incentivar a los inversionistas a rebalancear sus portafolios. Por ejemplo, al acumular 100 millones de dólares de reservas y esterilizar su impacto monetario, el Banco de México estaría aumentando la oferta de títulos en moneda nacional y aumentando la demanda por títulos en moneda extranjera por el mismo monto. En este caso, la teoría de portafolio predice que los inversionistas exigirán un rendimiento mayor sobre los activos en pesos para absorber su mayor oferta, induciendo una depreciación del tipo de cambio o un aumento en las tasas de interés. Por lo tanto, siempre que los activos nacionales y externos sean sustitutos imperfectos en los portafolios de los inversionistas, un cambio en su oferta relativa 
requerirá un cambio en los retornos esperados que se producirían a través de ajustes del tipo de cambio y/o de las tasas de interés internas.

La representación más sencilla del modelo de portafolio (Que se puede encontrar en los artículos de Branson (1979), Dornbusch (1983) y Frankel (1982).) extiende la ecuación de paridad de tasas de interés para tomar en cuenta la posibilidad de una prima de riesgo que depende de las ofertas de activos. Esta ecuación se presenta a continuación:

$$
i-i^{*}-\left(S_{t+1}^{e}-S_{t}\right)=f\left(B / B^{*}\right)
$$

En donde $i$ es la tasa de interés en moneda nacional, $i^{*}$ es la tasa de interés externa, $S_{t}$ es el logaritmo del tipo de cambio, ${ }^{S_{t+1}^{e}}$ es la esperanza del logaritmo del tipo de cambio en el período siguiente y $\left(B / B^{*}\right)$ es la razón de la oferta de bonos en moneda nacional a la oferta de bonos en moneda extranjera. Esta ecuación nos dice que a medida que la oferta de títulos nacionales $(B)$ aumenta, el retorno de éstos tendrá que aumentar. Este aumento se puede dar a través de un aumento en la tasa de interés en moneda nacional $(i)$ o a través de una disminución en la depreciación esperada. Para que se produzca una menor depreciación esperada de la moneda nacional, su cotización al contado se tiene que depreciar. Esta ecuación también puede interpretarse como una demanda de títulos denominados en moneda nacional. En esta interpretación tenemos que si aumenta el retorno de una inversión en estos títulos aumentará su demanda. Las estimaciones empíricas (Véase los estudios de Domínguez, Frankel y Edison.) de la ecuación (1) para países industrializados concluyen que el impacto de las ofertas relativas de bonos sobre el retorno esperado ha sido nulo (o en el mejor de los casos muy pequeño), por lo que una intervención esterilizada no tiene mayor efecto sobre el tipo de cambio o sobre las tasas de interés. Hay que destacar que en México el tamaño de las operaciones de esterilización en relación al acervo de títulos gubernamentales en moneda nacional ha sido mucho mayor a lo que se ha observado en los países industrializados. Por este motivo es posible que el impacto de estas operaciones pu diera ser mayor en México que el observado en dichos países. Otra razón para que estas operaciones tengan un efecto mayor en México, es que tal vez los activos en moneda nacional sean peores sustitutos de los demás activos en los portafolios de los inversionistas que los activos denominados en las monedas de los países desarrollados.

La ecuación (1) también puede derivarse para el caso en el que existen diferentes activos denominados en moneda nacional. Esta extensión puede servir para explicar la razón por la cual las tasas de interés de los títulos gubernamentales 
denominados en moneda nacional aumentan con la acumulación esterilizada de reservas internacionales. Esto ocurriría si el exceso de oferta de divisas que dispara la intervención del banco central en el mercado cambiario tiene como contrapartida un aumento en la demanda por títulos no gubernamentales denominados en moneda nacional. Si la autoridad monetaria realiza la operación de esterilización con títulos gubernamentales, el retorno de éstos tendría que aumentar y el de los títulos que experimentaron un aumento en su demanda se reducirá. Mientras el grado de sustitución entre estos dos activos sea menor los movimientos que se observarán en sus tasas de retorno serán mayores.

Para la evaluación empírica del modelo de portafolio se utilizará la ecuación (1) para estudiar los determinantes de las tasas de interés en moneda nacional. Para esta estimación se incluirá como una variable adicional que mide el riesgo México al diferencial entre la tasa de rendimiento de los bonos Brady emitidos por el país y las tasas de rendimiento de los bonos de los Estados Unidos a un plazo comparable.

b) Efecto Señal: El segundo mecanismo a través del cual una intervención esterilizada puede afectar el nivel del tipo de cambio y de las tasas de interés se conoce como el efecto señal, que no depende de los efectos de portafolio. De acuerdo a esta hipótesis, las intervenciones esterilizadas podrían tener un efecto sobre estas variables cuando dichas intervenciones sean usadas por los bancos centrales para transmitir información al mercado sobre el curso futuro de los fundamentos (por ejemplo, podrían señalar la posibilidad de una política más laxa en el futuro). Si los participantes del mercado creen que estas intervenciones tienen un efectos señal, aún cuando los fundamentos actuales no cambien, la expectativa de estos fundamentos en el futuro se afectará, ocasionando movimientos en el tipo de cambio y en las tasas de interés. Los estudios empíricos que se han realizado al respecto no son concluyentes, pero en algunos de ellos se ha encontrado evidencia en favor de este mecanismo (Además de los estudios anteriores, que también analizan el efecto señal, véase los trabajos de Mussa, Lewis y Kaminsky y Lewis.).

A continuación se hace una breve descripción del mecanismo de intervención en el mercado cambiario en México y la política de esterilización que se ha seguido, para posteriormente realizar una evaluación empírica de su impacto sobre el tipo de cambio y el nivel de tasas de interés. 


\section{II.1- Efectos de las intervenciones en el mercado de cambios a través del mecanismo de opciones.}

El régimen cambiario de libre flotación fue adoptado en México después de la devaluación de diciembre de 1994. Durante el primer año de la vigencia de este régimen, la cotización del peso frente al dólar mostró una gran inestabilidad, como resultado de un ambiente generalizado de incertidumbre macroeconómica. A partir del inicio de 1996, y al normalizarse la situación económica, dicha cotización mostró una reducción considerable en su volatilidad.

Durante la crisis cambiaria de 1994 los activos internacionales del Banco de México se redujeron considerablemente. Debido a esto, en agosto de 1996 se estimó conveniente por razones prudenciales, acelerar la acumulación de activos internacionales. Un mayor monto de éstos contribuiría a que el país obtuviese mejores condiciones en cuanto al costo y al plazo del financiamiento externo. Posteriormente, se ha apoyado el uso de la intervención mediante opciones con el objeto de evitar la apreciación de la moneda nacional. Hay que destacar que las expansiones de liquidez que han resultado de las compras de divisas por parte del Banco de México han sido esterilizadas en su totalidad.

En base a estas consideraciones, se estableció un programa de adquisición de divisas a través de la venta de opciones de compra de dólares por parte del propio Banco de México. Al inicio del programa, se decidió que las subastas mensuales de opciones serían de 130 millones de dólares. Este monto se aumentó inmediatamente a 200 millones de dólares para septiembre de 1996 y a 300 millones de dólares en diciembre de ese año. En febrero de 1997, la Comisión de Cambios resolvió que a partir de ese mes, en caso de ejercerse antes del día 16 el $80 \%$ o más de las opciones correspondientes a ese mes, se convocaría inmediatamente a una subasta adicional de opciones por otros 300 millones de dólares. Estas últimas tendrían vigencia sólo por el resto del mes respectivo (En esta fecha la Comisión de Cambios también anunció que de observarse una depreciación diaria mayor a 2\%, el Banco de México subastaría 200 m.d.d..). A finales de julio de 1997, la Comisión de Cambios decidió incrementar a 500 millones de dólares la subasta de opciones susceptibles de ejercerse en agosto, realizándose una subasta por igual cantidad si, durante la primera quincena, se hubieran ejercido el $80 \%$ o más de las opciones subastadas el 31 de julio. Este esquema continuó vigente durante septiembre. A partir de los ajustes realizados al programa monetario del Banco de México, el 29 de septiembre de este año se decidió disminuir el monto de opciones subastadas a 400 m.d.d.. Finalmente, el 30 de octubre estas opciones se redujeron nuevamente a 250 m.d.d.. 
El mecanismo de opciones ha cumplido con el principal objetivo para el cual fue creado. Durante los 16 meses de operación el Banco de México ha adquirido 5'095 millones de dólares a través del mecanismo. El resumen de estas compras se presenta en el cuadro 1. Sin embargo, su uso no ha sido tan exitoso en términos de evitar la apreciación de la moneda nacional. Evidencia al respecto se presenta más adelante. No sólo las intervenciones esterilizadas no han evitado la fortaleza de la moneda nacional en algunos períodos, sino que además hay cierta evidencia de que dichas intervenciones han redundado en tasas de interés internas ligeramente mayores.

\section{Cuadro 1}

\section{Compra de Dólares Mediante}

$$
\text { el Mecanismo de Opciones }
$$

\begin{tabular}{cccr}
\hline $\begin{array}{c}\text { Fecha } \\
\text { de la } \\
\text { Subasta }\end{array}$ & $\begin{array}{c}\text { Monto } \\
\text { Subastado } \\
\text { (MDDD) }\end{array}$ & $\begin{array}{c}\text { Monto } \\
\text { Ejercido } \\
\text { (MDD) }\end{array}$ & $\begin{array}{r}\text { Proporción } \\
\text { Ejercida }\end{array}$ \\
\hline 7-ago-96 & 130 & 130 & $100.0 \%$ \\
30-ago-96 & 200 & 200 & $100.0 \%$ \\
30-sep-96 & 200 & 179 & $89.5 \%$ \\
31-oct-96 & 200 & 200 & $100.0 \%$ \\
29-nov-96 & 200 & 200 & $100.0 \%$ \\
30-dic-96 & 300 & 300 & $100.0 \%$ \\
31-ene-97 & 300 & 300 & $100.0 \%$ \\
21-feb-97 & 300 & 148 & $49.3 \%$ \\
28-feb-97 & 300 & 120 & $40.0 \%$ \\
31-mar-97 & 300 & 300 & $100.0 \%$ \\
15-abr-97 & 300 & 263 & $87.7 \%$ \\
30-abr-97 & 300 & 300 & $100.0 \%$ \\
30-may $\mathbf{9 7}$ & 300 & 20 & $6.7 \%$ \\
30-jun-97 & 300 & 300 & $100.0 \%$ \\
9-jul-97 & 300 & 300 & $100.0 \%$ \\
31-jul-97 & 500 & 460 & $92.0 \%$ \\
29-ago-97 & 500 & 500 & $100.0 \%$ \\
30-sep-97 & 400 & 375 & $93.8 \%$ \\
31-oct-97 & 250 & 250 & $100.0 \%$ \\
28-nov-97 & 250 & 250 & $100.0 \%$ \\
Total & $\mathbf{5 8 3 0}$ & $\mathbf{5 0 9 5}$ & $\mathbf{8 7 . 4} \%$ \\
\hline & & & \\
\hline & & &
\end{tabular}


A continuación se hace una evaluación empírica del impacto de las intervenciones esterilizadas sobre el nivel del tipo de cambio y el nivel de las tasas de interés.

Para evaluar el efecto del programa de acumulación de activos internacionales en el comportamiento del tipo de cambio y de las tasas de interés, primero se realizan dos comparaciones del proceso estocástico seguido por estas variables. En primer lugar, comparamos estos procesos estocásticos antes de la adopción del programa de acumulación de reservas con los que se han observado a partir de la implementación de dicho programa. En segundo lugar, comparamos el proceso seguido por el tipo de cambio y las tasas de interés a partir de la implementación de este programa en los días en los cuales aún hay opciones no ejercidas, con el proceso seguido en los días en que no hay opciones no ejercidas, por lo que la probabilidad de intervenciones es prácticamente cero.

Se procedió a estimar el proceso estocástico para el tipo de cambio en función del diferencial de tasas de los bonos Brady (como una medida de riesgo país) y de las tasas de interés internacionales y nacionales. Se trató de detectar a la vez los posibles cambios en los procesos dependiendo de si se ha implementado o no el mecanismo de acumulación de activos internacionales y de si hay opciones sin ejercerse o no. Se realizó un ejercicio similar para las tasas de interés, en cuyo caso se utilizaron como variables independientes a las tasas de interés externas, las expectativas de devaluación y el diferencial de tasas de los bonos Brady. Como ya se ha demostrado en un trabajo anterior (Werner (1997)), el proceso estocástico seguido por el tipo de cambio del peso en el régimen de flotación se puede caracterizar por una caminata al azar con tendencia. En ciertos períodos, el diferencial de tasas de los bonos Brady y las tasas de interés interna y externa contribuyen marginalmente a explicar las fluctuaciones cambiarias. De ahí que se incorporen en las regresiones estimadas.

Las ecuaciones utilizadas son las siguientes:

$$
\begin{aligned}
\Delta S_{t} & =B_{0}+B_{1} D U M_{t}+B_{2} i_{t}^{*}+B_{3} i_{t-1}^{*}+B_{4}^{i_{t-1}}+B_{g_{t-2}}^{i_{t}}+B_{6} S P_{t} \\
& +B_{7} S P_{t-1}+B_{8} S P_{t-2}
\end{aligned}
$$




$$
\begin{aligned}
i_{t}= & B_{0}+B_{1} D U M_{t}+B_{2} i_{t}^{*}+B_{3} i_{t-1}^{*}+B_{4} E D E V_{t}+B_{5} E D E V_{t-1}+B_{6} E D E V_{t-2} \\
& +B_{7_{t-1}}+B_{8} i_{t-2}+B_{9} i_{t-3}+B_{10} S P_{t}+B_{11} S P_{t-1}+B_{12} S P_{t-2}
\end{aligned}
$$

En la ecuación (2), $\Delta S_{\text {tes }}$ la depreciación del tipo de cambio, ${ }_{i_{t}^{*}}^{*}$ es el retorno del bono a 30 años del Tesoro de los Estados Unidos, ${ }^{i_{t}}$ es la tasa de fondeo en pesos (Las regresiones de esta nota también se estimaron utilizando la tasa de interés a 3 meses, lo cual no afectó ninguno de los resultados que aquí se presentan.) y $S P_{t}$ es el diferencial de tasas de los bonos Brady. En la ecuación (3), EDEV es la expectativa de depreciación del tipo de cambio que se mide a través del precio de las coberturas cambiarias. Estas ecuaciones se estiman primero para el período enero 1996 a diciembre de 1997; en este caso la variable $D U M$ es una variable binaria que toma el valor de 1 para el período agosto 1996-diciembre 1997 y 0 para el período enero-julio de 1996 (con esto se trata de capturar la importancia de la introducción del mecanismo de opciones). Posteriormente, estas ecuaciones se estiman para el período agosto 1996-diciembre 1997; en este caso la variable $D U M$ toma el valor de 1 en los días en los que aún no se ha ejercido la totalidad de las opciones y 0 para los días en los que ya no hay opciones vigentes. Si las intervenciones en el mercado cambiario contribuyen a depreciar al tipo de cambio y a aumentar las tasas de interés, esto se reflejaría en que el coeficiente asociado a la variable DUM sería positivo y significativo. Por otro lado, los coeficientes asociados a las tasas de interés externas y al diferencial de tasas de los bonos Brady deben ser positivos, ya que un aumento de estas variables conduciría a una depreciación del tipo de cambio y a un aumento en las tasas de interés en pesos (Debido a que las pruebas de raíz unitaria para las variables $i, i^{*}$ y $S P$ no fueron concluyentes, todas las regresionesse reestimaron usando las diferencias de estas variables y los resultados obtenidos fueron similares a los que se presentan en la nota.). También se espera que en la ecuación (2) un aumento de la tasa de interés en pesos conduzca a una apreciación del tipo de cambio, por lo que sus coeficientes deben ser negativos. Finalmente, en la ecuación (3), los coeficientes asociados a las expectativas de depreciación $(E D E V)$ deben ser positivos, ya que un aumento de éstas se reflejará en tasas de interés más altas.

En los cuadros 2 y 3 se presentan los resultados de las estimaciones de las ecuaciones (2) y (3), respectivamente. 


\section{Cuadro 2}

\section{Tipo de Cambio}

\begin{tabular}{|c|c|c|c|c|c|c|c|c|c|c|}
\hline & $\boldsymbol{B}_{0}$ & $B_{1}$ & $\boldsymbol{B}_{2}$ & $\boldsymbol{B}_{3}$ & $B_{4}$ & $B_{5}$ & $B_{6}$ & $\boldsymbol{B}_{7}$ & $\boldsymbol{B}_{8}$ & $R^{2}$ \\
\hline \multirow{2}{*}{$\begin{array}{r}\text { Ene } 1996 \\
\text { Dic } 1997^{1 /}\end{array}$} & -0.0001 & 0.0007 & 0.003 & -0.003 & -0.0004 & 0.0003 & 0.001 & \begin{tabular}{|l}
-0.008 \\
\end{tabular} & \begin{tabular}{|l}
$\mid-0.002$ \\
\end{tabular} & 0.29 \\
\hline & $(-0.21)$ & (1.36) & $(0.66)$ & $(-0.69)$ & $(-2.71)$ & (1.82) & (3.23) & $(-4.01)$ & $(-1.17)$ & \\
\hline \multirow{2}{*}{$\begin{array}{r}\text { Ago } 1996 \\
\text { Dic } 1997^{2 /}\end{array}$} & 0.0001 & -0.0005 & 0.008 & -0.008 & -0.0005 & 0.0004 & 0.018 & -0.010 & -0.007 & 0.47 \\
\hline & $(0.02)$ & $(-1.45)$ & (1.88) & $(-1.98)$ & $(-2.81)$ & (1.91) & (4.66) & $(-5.03)$ & $(-2.98)$ & \\
\hline
\end{tabular}

estadísticos $t$ entre paréntesis

1/ En este caso la variable DUM toma el valor de 1 para el período en el cual se implementó el programa de opciones (Agosto 1996-Septiembre 1997) y 0 para el período Enero-Agosto de 1996.

2/ En este caso la variable DUM toma el valor de 1 los días en los cuales aún hay opciones vigentes y 0 en los días en los cuales éstas se han ejercido en su totalidad. 


\section{Cuadro 3}

\section{Tasas de Interés}

\begin{tabular}{|c|c|c|c|c|c|c|c|c|c|c|c|c|c|c|}
\hline & $\boldsymbol{B}_{0}$ & $B_{1}$ & $\boldsymbol{B}_{2}$ & $\boldsymbol{B}_{3}$ & $B_{4}$ & $B_{5}$ & $B_{6}$ & $\boldsymbol{B}_{7}$ & $\boldsymbol{B}_{8}$ & $\boldsymbol{B}_{9}$ & $B_{10}$ & $B_{11}$ & $B_{12}$ & $R^{2}$ \\
\hline \multirow{2}{*}{$\begin{array}{r}\text { Ene } 1996 \\
\text { Dic } 1997^{1 /}\end{array}$} & 2.84 & 0.09 & 0.86 & -1.19 & 379.83 & -153.0 & -94.99 & 0.92 & -0.14 & 0.06 & 0.80 & 0.18 & -0.84 & 0.98 \\
\hline & (2.40) & $(0.63)$ & (1.10) & $(-1.53)$ & (6.50) & $(-2.39)$ & $(-2.24)$ & (19.08) & $(-2.13)$ & (1.48) & (3.75) & $(0.47)$ & $(-3.37)$ & \\
\hline \multirow{2}{*}{$\begin{array}{r}\text { Ago } 1996 \\
\text { Dic } 1997^{2 /}\end{array}$} & 5.31 & 0.24 & 0.56 & -1.26 & 343.71 & -67.18 & -75.92 & 0.87 & -0.16 & 0.08 & 0.66 & 0.55 & -1.11 & 0.97 \\
\hline & (3.38) & (3.04) & $(0.72)$ & $(-1.48)$ & (5.63) & $(-1.06)$ & $(-1.85)$ & $(16.51)$ & $(-2.15)$ & (1.64) & (3.63) & (1.66) & $(-4.23)$ & \\
\hline
\end{tabular}

estadísticos $t$ entre paréntesis

1/ En este caso la variable DUM toma el valor de 1 para el período en el cual se implementó el programa de opciones (Agosto 1996Septiembre 1997) y 0 para el período Enero-Agosto de 1996.

2/ En este caso la variable DUM toma el valor de 1 los días en los cuales aún hay opciones vigentes y 0 en los días en los cuales éstas se han ejercido en su totalidad.

El bajo nivel de las R2 en las ecuaciones que explican las fluctuaciones cambiarias indica que la contribución de las tasas de interés, interna y externa, del diferencial de tasas en los bonos Brady y de las variables dummies en la explicación de las fluctuaciones cambiarias es relativamente baja. Sin embargo, el objetivo del ejercicio no es determinar el mejor modelo para explicar el comportamiento del tipo de cambio, sino estudiar el impacto que tienen ciertos fenómenos sobre el tipo de cambio. El resultado de estas estimaciones confirma que el proceso seguido por el tipo de cambio no se vió alterado por la introducción del mecanismo de acumulación de reservas en agosto de 1996, ya que en el cuadro 2 los coeficientes asociados a la variable $\operatorname{DUM}\left(B_{1}\right)$ no son estadísticamente significativos. 
La estimación de la ecuación que explica el comportamiento de la tasa de interés de fondeo sugiere que ésta aparentemente no se ha visto afectada por la introducción del mecanismo de las opciones; sin embargo, los resultados también indican que dicha tasa ha sido ligeramente más elevada ( 24 puntos base) en los días en los que aún existen opciones sin ejercer, que en los días en los que estas opciones se ha ejercido totalmente. Si el proceso de esterilización ejerce una presión al alza en las tasas de interés y los agentes económicos anticipan este efecto, el régimen de saldos acumulados (En este régimen el Banco de México no exige que las instituciones de crédito constituyan reservas en el instituto emisor. Sin embargo, bajo este esquema la autoridad monetaria exige a las instituciones de crédito que durante un período determinado el saldo promedio diario de sus cuentas en el banco central sea cero. Para una explicación más detallada de este esquema consulte el apéndice 4 del Informe Anual de 1996 y el volumen La Política Monetaria en México (ITAM).) podría contribuir a que este efecto se refleje de manera anticipada en las tasas de interés. Es pertinente recordar que una de las propiedades del régimen de saldos acumulados es que permite el arbitraje de tasas de interés entre los momentos en los que exista presión al alza sobre éstas y los momentos en los que dicha presión no existe. Por lo tanto, se observaría que en aquellos períodos en los cuales existe la posibilidad que la autoridad tenga que realizar operaciones de esterilización las tasas de interés serían ligeramente más elevadas. Los resultados econométricos parecen avalar esta hipótesis.

A continuación se analiza si el ejercicio de estas opciones tiene algún efecto en los mercados cambiario y de dinero. Para esto suponemos que el tipo de cambio se determina de acuerdo a la siguiente ecuación:

$$
\begin{aligned}
\Delta S_{t}= & B_{0}+B_{1^{i} t}^{i^{*}}+B_{2} i_{t-1}^{*}+B_{\xi_{t-1}^{i}}+B_{4_{t-2}^{i}}+B_{5} \text { Intervención } \\
& +B_{6} S P_{t}+B_{7} S P_{t-1}+B_{8} S P_{t-2}+B_{7} S P_{t-3}
\end{aligned}
$$

En donde Intervención $n_{t}$ es el monto ejercido de las opciones durante el día $t$. Las tasas de interés a su vez, se determinan de acuerdo a la siguiente ecuación:

$$
\begin{aligned}
\dot{i}_{t} & =B_{0}+B_{1^{i_{t}}} \dot{\vec{t}}^{*}+B_{2} \dot{i}_{t-1}^{\dot{0}}+B_{3} E D E V_{t}+B_{4} E D E V_{t-1} \\
& +B_{5} \text { Intervención } n_{t}+B_{6} S P_{t}+B_{7} S P_{t-1}+B_{8} i_{t-1}+B_{9} i_{t-2}
\end{aligned}
$$


Es pertinente apuntar, que Intervención no es una variable exógena ya que los dueños de las opciones deciden ejercerlas en los días de mayor apreciación. Debido a esto, la variable Intervención estará correlacionada con el error de la ecuación y los estimadores serán inconsistentes. Para resolver este problema, se estiman la ecuaciones (4) y (5) a través del método de variables instrumentales, usando como instrumento la diferencia entre el tipo de cambio del día anterior y el promedio móvil de los 20 días anteriores. Solamente cuando esta variable es negativa se puede ejercer la opción. Por lo tanto, al estar correlacionada con Intervención y no con el cambio en el tipo de cambio es un instrumento válido. Como las operaciones de compra y venta de dólares se realizan fecha valor 48 horas, otra manera de atacar este problema sería estimando esta ecuación con el valor rezagado (en dos períodos) de la variable Intervención. Como se verá en los resultados, los dos métodos arrojan resultados similares.

En el cuadro 4 se presentan los resultados de estas regresiones. Las estimaciones se efectuaron para todo el período en el que han estado vigentes las opciones y también se dividió este período en dos subperíodos, correspondientes a los años 1996 y 1997. Para cada período se presentan resultados de los tres métodos mencionados: mínimos cuadrados ordinarios (MCO), variables instrumentales (VI) y mínimos cuadrados ordinarios con la variable intervención rezagada (REZAGOS). En la ecuación que explica los cambios en el tipo de cambio, cuando se estima a través del método de mínimos cuadrados, el coeficiente de la variable Intervención $\left(\mathrm{B}_{3}\right)$ resulta significativo pero negativo. Este resultado indica que este coeficiente está capturando la causalidad inversa, ya que cuando la apreciación es mayor es más rentable ejercer la opción y la variable Intervención será mayor. Esta interpretación se confirma ya que en los resultados de la estimación con variables instrumentales y con la variable rezagada, el coeficiente de la variable intervención en algunos casos se vuelve positivo pero no es significativo (Aunque para el período enero-diciembre 1997, el coeficiente es significativo al 15\% de confianza.). Este resultado indica que una vez que se aisla el efecto que va de una mayor apreciación a un mayor monto de opciones ejercido, la intervención no tiene un impacto importante sobre el tipo de cambio.

La $\mathrm{R}^{2}$ de las regresiones estimadas por variables instrumentales son negativas debido a la baja correlación entre la variable a instrumentar y la variable instrumental utilizada. Se intentaron otros instrumentos sin que los resultados aquí presentados se hayan visto alterados.

En los resultados de la regresión que estudia el comportamiento de la tasa de fondeo no se encuentra ningún efecto significativo de la intervención diaria sobre esta tasa, ya que en el cuadro 5 el coeficiente asociado a la variable Intervención $\left(\mathrm{B}_{5}\right)$ no es estadísticamente significativo. Dado los resultados presentados en el 
cuadro 3, la falta de una relación contemporánea entre las intervenciones y los aumentos en las tasas de interés puede deberse a que el mercado anticipa la posibilidad de una intervención, por lo que éstas aumentan con anticipación y se mantienen en este nivel más elevado mientras existan opciones sin ejercerse.

\section{Cuadro 4}

$\Delta S_{t}=B_{0}+B_{1_{t}^{i}}^{*}+B_{2^{i} i_{t-1}^{*}}+B_{3} i_{t-1}+B_{4} i_{t-2}+B_{5}$ Intervención $+B_{6} S P_{t}+B_{7} S P_{t-1}+B_{8} S P_{t-2}+B_{7} S P_{t-3}$

\begin{tabular}{|c|c|c|c|c|c|c|c|c|c|c|c|}
\hline Método & $\mathbf{B}_{0}$ & $\mathbf{B}_{1}$ & $\mathbf{B}_{2}$ & $\mathbf{B}_{3}$ & $\mathbf{B}_{4}$ & $\mathbf{B}_{5}$ & $\mathbf{B}_{6}$ & $\mathbf{B}_{7}$ & $\mathbf{B}_{8}$ & $\mathbf{B}_{9}$ & $\mathbf{R}^{2}$ \\
\hline \multicolumn{12}{|c|}{ Agosto 96 - Diciembre 97} \\
\hline \multirow[t]{2}{*}{ MCO } & -0.0002 & 0.008 & -0.008 & -0.0007 & 0.0006 & $-6.8 \mathrm{E}-06$ & 0.018 & -0.009 & -0.005 & -0.002 & 0.47 \\
\hline & $(-0.04)$ & (1.93) & $(-2.01)$ & $(-3.57)$ & $(2.79)$ & $(-1.97)$ & $(4.68)$ & $(-4.75)$ & $(-2.08)$ & $(-2.24)$ & \\
\hline \multirow[t]{2}{*}{ VI } & -0.005 & 0.016 & -0.016 & -0.0007 & 0.0005 & $9.80 \mathrm{E}-05$ & 0.020 & -0.010 & -0.005 & -0.003 & -0.25 \\
\hline & $(-0.61)$ & (1.95) & $(-1.96)$ & $(-1.85)$ & $(1.20)$ & $(1.68)$ & $(4.22)$ & $(-4.50)$ & $(-1.91)$ & $(-2.18)$ & \\
\hline \multirow[t]{2}{*}{ REZAGO } & -0.001 & 0.008 & -0.008 & -0.0007 & 0.0005 & 8.28E-06 & 0.018 & -0.009 & -0.005 & -0.002 & 0.48 \\
\hline & $(-0.20)$ & (1.94) & $(-2.00)$ & $(-3.35)$ & $(2.57)$ & $(1.80)$ & $(4.69)$ & $(-4.71)$ & $(-2.12)$ & $(-2.13)$ & \\
\hline \multicolumn{12}{|c|}{ Agosto 96 - Diciembre 96} \\
\hline MCO & 0.010 & 0.012 & -0.013 & -0.0008 & 0.0006 & $-6.6 \mathrm{E}-06$ & 0.014 & -0.013 & 0.002 & -0.002 & 0.38 \\
\hline & $(0.55)$ & $(2.32)$ & $(-2.33)$ & $(-2.26)$ & (1.54) & $(-0.77)$ & $(4.52)$ & $(-3.05)$ & $(0.73)$ & $(-1.12)$ & \\
\hline & & & & & & & & & & & \\
\hline
\end{tabular}




\begin{tabular}{|c|c|c|c|c|c|c|c|c|c|c|c|}
\hline \multirow[t]{2}{*}{ VI } & 0.064 & 0.007 & -0.014 & -0.001 & 0.0003 & -0.0001 & 0.011 & -0.013 & 0.006 & -0.004 & -1.07 \\
\hline & $(0.93)$ & $(0.49)$ & $(-1.32)$ & $(-1.84)$ & $(0.56)$ & $(-0.94)$ & $(3.01)$ & $(-2.81)$ & $(1.00)$ & $(-1.26)$ & \\
\hline \multirow[t]{2}{*}{ REZAGO } & 0.007 & 0.012 & -0.012 & -0.0008 & 0.0006 & $6.74 \mathrm{E}-06$ & 0.014 & -0.013 & 0.002 & -0.002 & 0.38 \\
\hline & $(0.32)$ & $(2.32)$ & $(-2.21)$ & $(-2.30)$ & $(1.68)$ & $(0.91)$ & (4.60) & $(-3.04)$ & $(0.66)$ & $(-1.13)$ & \\
\hline \multicolumn{12}{|c|}{ Enero 97 - Diciembre 97} \\
\hline \multirow[t]{2}{*}{ MCO } & -0.001 & 0.007 & -0.006 & -0.0007 & 0.0004 & $-6.5 \mathrm{E}-06$ & 0.019 & -0.009 & -0.007 & -0.002 & 0.53 \\
\hline & $(-0.18)$ & (1.32) & $(-1.20)$ & $(-2.80)$ & $(1.50)$ & $(-1.47)$ & (4.33) & $(-3.77)$ & $(-2.80)$ & $(-2.39)$ & \\
\hline \multirow[t]{2}{*}{ VI } & -0.008 & 0.021 & -0.019 & -0.0009 & 0.0002 & 0.0001 & 0.021 & -0.009 & -0.006 & -0.004 & -0.33 \\
\hline & $(-0.73)$ & (1.57) & $(-1.47)$ & $(-1.71)$ & $(0.38)$ & (1.78) & (3.77) & $(-3.51)$ & $(-2.19)$ & $(-2.49)$ & \\
\hline \multirow[t]{2}{*}{ REZAGO } & -0.001 & 0.008 & -0.006 & -0.0006 & 0.0003 & 8.24E-06 & 0.019 & -0.008 & -0.007 & -0.002 & 0.53 \\
\hline & $(-0.33)$ & (1.37) & $(-1.24)$ & $(-2.58)$ & (1.23) & (1.53) & $(4.32)$ & $(-3.70)$ & $(-2.82)$ & $(-2.49)$ & \\
\hline & & & & & & & & & & & \\
\hline
\end{tabular}




\section{Cuadro 5}

$i_{t}=B_{0}+B_{1_{t}^{i}} \dot{*}^{+}+B_{2} i_{t-1}^{*}+B_{3} E D E V_{t}+B_{4} E D E V_{t-1}+B_{5}$ Intervencion $_{t}+B_{6} S P_{t}+B_{7} S P_{t-1}+B_{8} i_{t-1}+B_{9} i_{t-2}$

\begin{tabular}{|c|c|c|c|c|c|c|c|c|c|c|c|}
\hline Método & $\mathbf{B}_{0}$ & $\mathbf{B}_{1}$ & $\mathbf{B}_{2}$ & $\mathbf{B}_{3}$ & $\mathbf{B}_{4}$ & $\mathbf{B}_{5}$ & $\mathbf{B}_{6}$ & $\mathbf{B}_{7}$ & $\mathbf{B}_{8}$ & $\mathbf{B}_{9}$ & $\mathbf{R}^{2}$ \\
\hline \multicolumn{12}{|c|}{ Agosto 96 - Diciembre 97} \\
\hline \multirow[t]{2}{*}{ MCO } & 5.83 & 0.59 & -1.29 & 309.41 & -108.26 & -0.002 & 0.44 & -0.312 & 0.93 & -0.14 & 0.96 \\
\hline & (3.82) & $(0.72)$ & $(-1.50)$ & $(4.92)$ & $(-1.67)$ & $(-2.56)$ & (1.40) & $(-0.97)$ & (15.36) & $(-2.74)$ & \\
\hline \multirow[t]{2}{*}{ VI } & 5.81 & 0.566 & -1.26 & 308.54 & -108.91 & -0.002 & 0.43 & -0.30 & 0.93 & -0.14 & 0.96 \\
\hline & (3.69) & $(0.55)$ & $(-1.15)$ & $(4.98)$ & $(-1.63)$ & $(-0.37)$ & $(1.50)$ & $(-1.03)$ & (14.82) & $(-2.84)$ & \\
\hline \multirow[t]{2}{*}{ REZAGO } & 5.86 & 0.70 & -1.42 & 312.46 & -105.24 & 0.0003 & 0.47 & -0.33 & 0.93 & -0.15 & 0.96 \\
\hline & (1.40) & $(0.25)$ & $(-0.51)$ & $(4.96)$ & $(-1.16)$ & $(0.75)$ & $(2.21)$ & $(-2.45)$ & (16.38) & $(-4.35)$ & \\
\hline \multicolumn{12}{|c|}{ Agosto 96 - Diciembre. 96} \\
\hline \multirow[t]{2}{*}{ MCO } & 4.24 & -0.010 & -0.52 & 368.69 & -185.29 & $5.66 \mathrm{E}-05$ & 1.17 & -1.18 & 1.070 & -0.22 & 0.96 \\
\hline & $(1.39)$ & $(-0.007)$ & $(-0.38)$ & $(4.21)$ & $(-3.63)$ & $(0.029)$ & (2.11) & $(-2.31)$ & $(10.0)$ & $(-2.44)$ & \\
\hline \multirow[t]{2}{*}{ VI } & 22.11 & -1.96 & -0.68 & 274.63 & -165.13 & -0.05 & 0.61 & -0.42 & 1.01 & -0.27 & 0.69 \\
\hline & $(0.96)$ & $(-0.40)$ & $(-0.20)$ & $(1.82)$ & $(-1.15)$ & $(-1.01)$ & $(0.55)$ & $(-0.37)$ & $(5.88)$ & $(-1.91)$ & \\
\hline & & & & & & & & & & & \\
\hline
\end{tabular}




\begin{tabular}{|l|l|l|l|l|l|l|l|l|l|l|l|}
\hline & & & & & & & & & & & \\
\hline REZAGO & 3.89 & -0.066 & -0.42 & 367.99 & -184.47 & 0.001 & 1.20 & -1.20 & 1.06 & -0.21 & 0.96 \\
\hline & $(1.41)$ & $(-0.04)$ & $(-0.31)$ & $(4.23)$ & $(-3.54)$ & $(0.56)$ & $(2.10)$ & $(-2.29)$ & $(10.04)$ & $(-2.38)$ & \\
\hline & & & & & & & & & & & \\
\hline
\end{tabular}

Por último se intentó capturar por separado el efecto portafolio y el efecto señal. A continuación se presenta una breve descripción de las estimaciones realizadas y de los resultados obtenidos.

a) Efecto Portafolio: Partiendo de la ecuación (1), en la que se describió el efecto portafolio, se procedió a relacionar la oferta de valores gubernamentales en moneda nacional con el diferencial cubierto de tasas de interés. Si linealizamos dicha ecuación y suponemos que la oferta de títulos en moneda extranjera se mantiene constante, obtenemos la siguiente expresión: 


$$
i_{t}-i_{t}^{*}-\left(S_{t+1}^{e}-S_{t}\right)=C_{0}+C_{1} B_{t}
$$

En donde $B_{\mathrm{t}}$ es la oferta de títulos gubernamentales en moneda nacional y $S_{t+1}^{e}-S_{t}$ es la depreciación esperada del tipo de cambio que se mide a partir del precio de las coberturas cambiarias.

A partir de la ecuación (6) podemos obtener una ecuación que explica las fluctuaciones de la tasa de interés en moneda nacional en función de la tasa de interés externa, las expectativas de depreciación y el stock de títulos en moneda nacional. Esta ecuación se extiende a continuación para tomar en cuenta el riesgo México, medido a través del diferencial de tasas de los bonos Brady, y se incorpora una estructura de rezago más rica. Realizando estas modificaciones llegamos a la ecuación (7).

$$
\begin{aligned}
& i_{t}=B_{0}+B_{1} i_{t}^{*}+B_{2} i_{t-1}^{*}+B_{3} E D E V_{t}+B_{4} E D E V_{t-1}+B_{5} S P_{t} \\
& +B_{6} S P_{t-1}+B_{7} S P_{t-2}+B_{8} \ln C E T E S_{t}+B_{9} i_{t-1}+B_{10 t_{t-2}^{i}}+B_{11^{i} t-3}
\end{aligned}
$$

En donde InCETES es el logaritmo natural del acervo de CETES en poder del sector privado. Dado que el coeficiente de lnCETES $\left(\mathrm{B}_{8}\right)$ no puede distinguir entre la relación que existe entre la tasa de interés interna y la cantidad de títulos en moneda nacional sobre la curva de demanda de la relación que existe sobre la curva de oferta de títulos, la ecuación (7) se estimó con MCO y VI. La estimación a través de variables instrumentales captura únicamente la relación de demanda ( $\mathrm{Se}$ utilizaron variables dummies semanales y el rezago del stock de CETES como variables instrumentales.). La ecuación se estimó con datos diarios para el período de enero de 1996 a diciembre de 1997 (Como la variable InCETES es no estacionaria, se utilizó la primera diferencia de esta variable en las regresiones estimadas.). En los resultados se observa que solamente para la muestra juliodiciembre de 1997 se obtiene, para la oferta de títulos en moneda nacional, un coeficiente positivo y significativo, que es lo que se necesita como evidencia de que una intervención esterilizada afecta a las tasas de interés. Por lo tanto, para el período julio a diciembre de 1997 se detectó un efecto positivo y significativo del acervo de títulos en moneda nacional sobre las tasas de interés. Como para el resto del período no se detectó ningún efecto, este resultado se puede interpretar como una señal de que en la medida que el acervo de títulos en moneda nacional ha aumentado, las operaciones de esterilización han comenzado a tener un impacto reducido sobre las tasas de interés. 


\section{Cuadro 6}

$i_{t}=B_{0}+B_{1} i_{t}^{*}+B_{2} i_{t-1}^{*}+B_{3} E D E V_{t}+B_{4} E D E V_{t-1}+B_{5} S P_{t}+B_{6} S P_{t-1}+B_{7} S P_{t-2}+B_{8} \Delta \ln C E T E S_{t}+B_{9} i_{t-1}+B_{10} i_{t-2}+B_{11^{i} t-3} i_{t-1}$

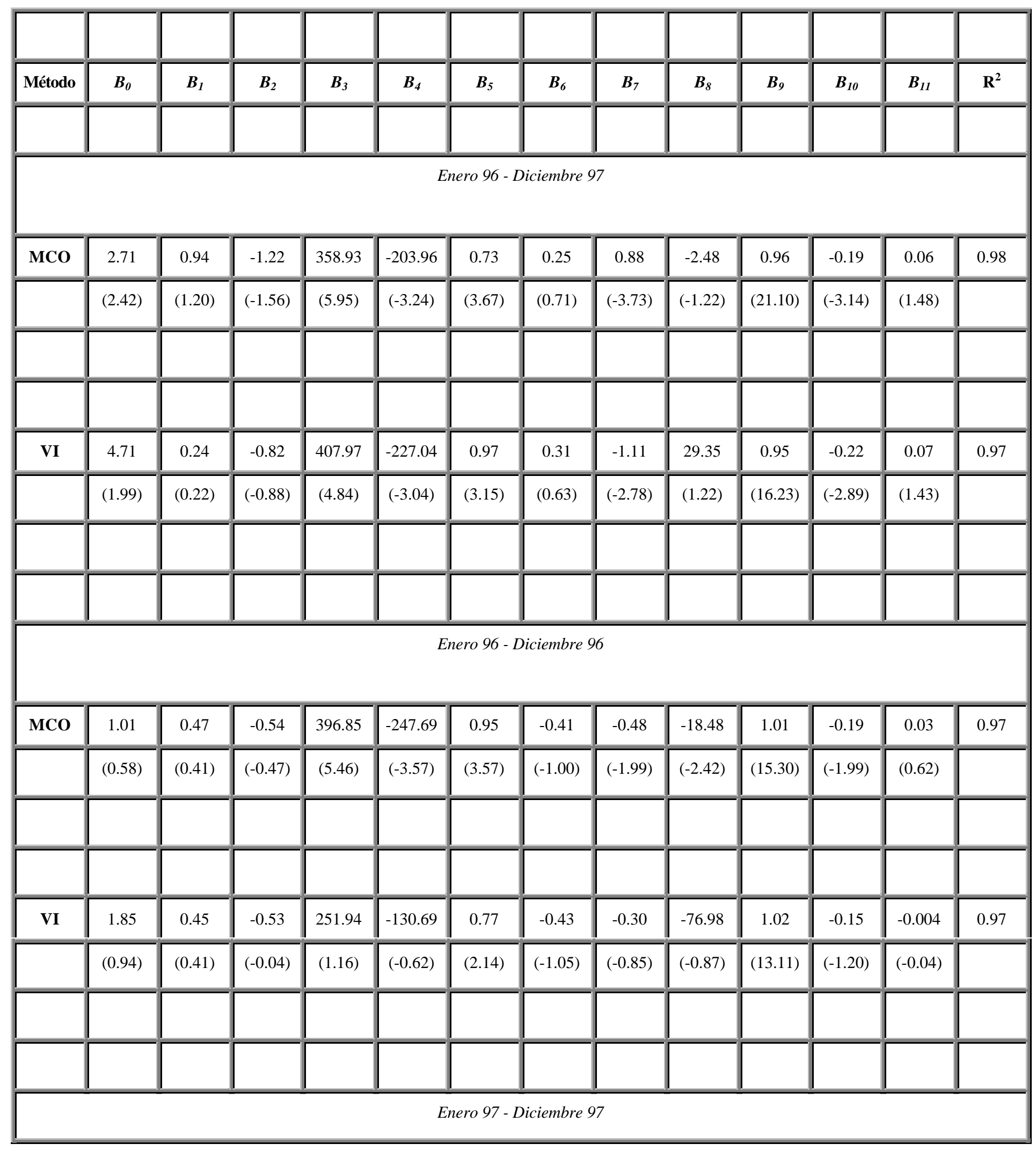




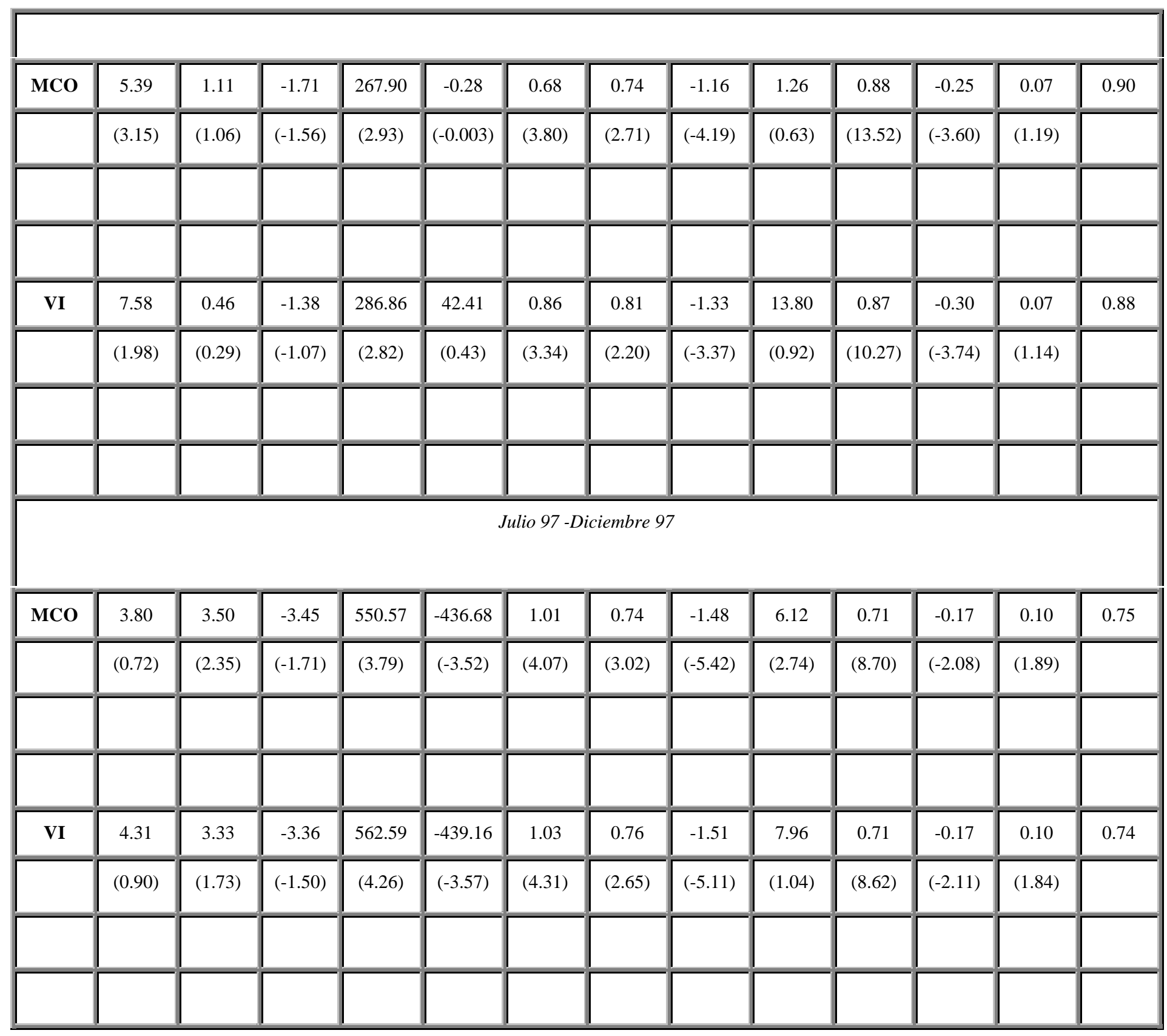

b) Efecto Señal: Dado que este efecto operaría a través de modificaciones en las expectativas, es necesario analizar el proceso de formación de éstas. Dicho proceso se puede representar mediante la siguiente ecuación:

(8) $E D E V_{t}=\gamma_{0}+\gamma_{1} D E V_{t}+\gamma_{2} E D E V_{t-1}+\gamma_{3}$ Intervenciónt 
En donde $E D E V_{\mathrm{t}}$ es la expectativa de depreciación mensual a partir del período $t$ medida mediante el precio de las coberturas y $D E V_{t}$ es la depreciación diaria observada entre $t$ y $t$ - 1 . Esta especificación nos indica que las expectativas de depreciación se forman a partir de las expectativas del período pasado, de la devaluación observada en el período presente y de la posible señal sobre los fundamentos futuros que emiten las autoridades al momento de intervenir en el mercado cambiario.

Una especificación alternativa para la ecuación de formación de expectativas sostiene que la revisión de las expectativas de devaluación depende de los cambios en la devaluación observada ${ }^{(\triangle D e v)}$ y de la diferencia entre la devaluación observada y la que se esperaba para ese período. Haciendo estos supuestos se obtiene:

$$
\triangle\left(E D E V_{\mathrm{t}}\right)=\gamma_{0}+\gamma_{1} \Delta\left(D E V_{t}\right)+\gamma_{2}\left(D E V_{t-1}-E D E V_{t-1}\right)+\gamma_{3} \text { Intervención } n_{t}
$$

Estas ecuaciones que explican la formación de las expectativas de devaluación se estiman a continuación. Si las intervenciones son interpretadas por el mercado como una señal de un relajamiento en la política monetaria, éstas tenderían a aumentar las expectativas de devaluación $\left(\gamma_{3}>0\right)$. Por otro lado, se espera que los coeficientes asociados a la devaluación observada y al rezago de las expectativas de depreciación sean positivos, ya que un aumento en estas variables conducirá a un aumento en las expectativas de devaluación en el período presente. 


\section{Cuadro 7}

$E D E V_{t}=\gamma_{0}+\gamma_{1} D E V_{t}+\gamma_{2} E D E V_{t-1}+\gamma_{3}$ Intervencion $_{t}$

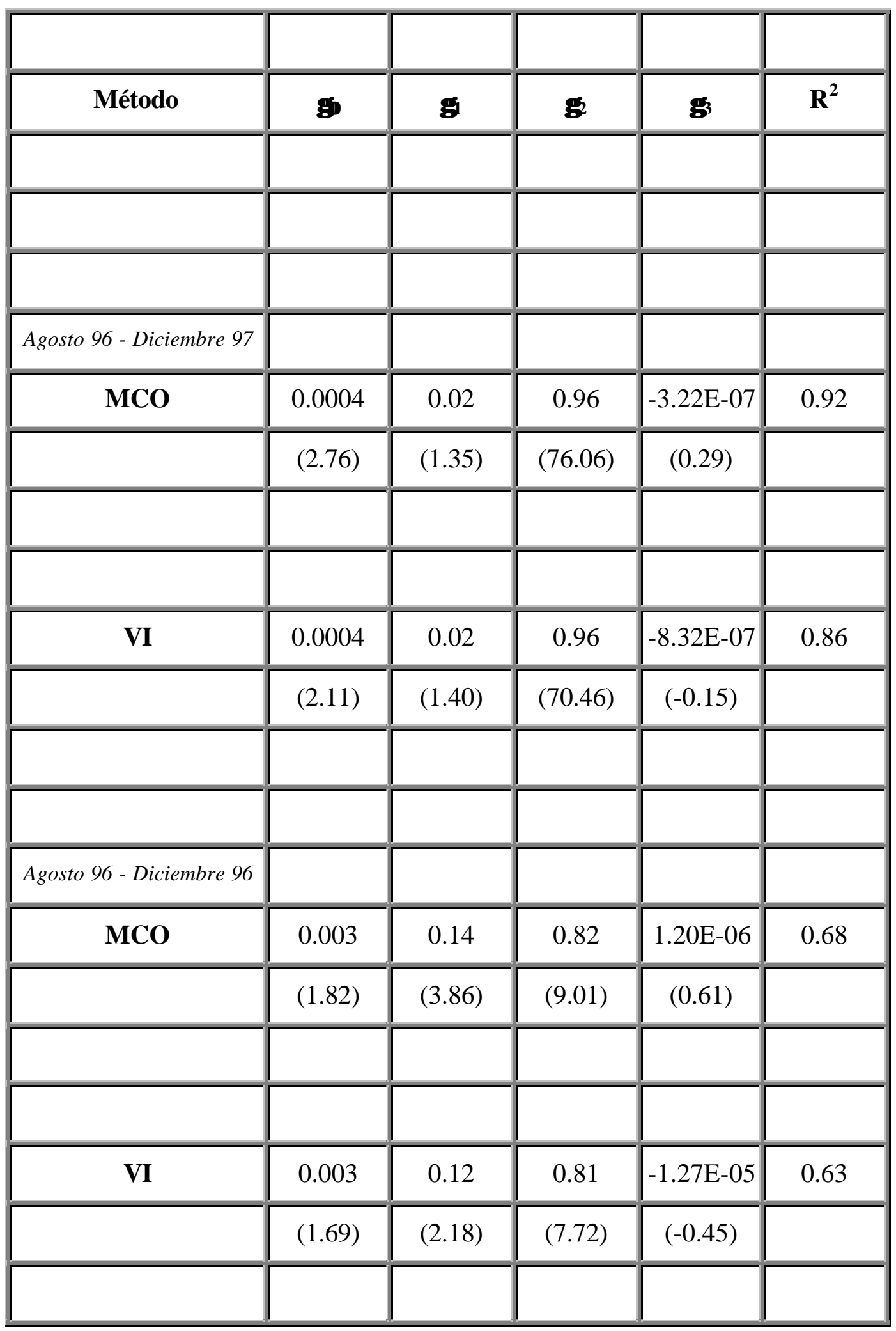




\begin{tabular}{|c|c|c|c|c|c|}
\hline & & & & & \\
\hline Enero 97 - Diciembre 97 & & & & & \\
\hline MCO & 0.0008 & -0.0006 & 0.93 & $-3.83 \mathrm{E}-07$ & 0.87 \\
\hline & $(2.79)$ & $(-0.08)$ & $(38.44)$ & $(-0.40)$ & \\
\hline & & & & & \\
\hline & & & & & \\
\hline & 0.0007 & 0.002 & 0.93 & $2.71 \mathrm{E}-06$ & 0.87 \\
\hline VI & $(2.60)$ & $(0.34)$ & $(38.24)$ & $(0.63)$ & \\
\hline & & & & & \\
\hline & & & & & \\
\hline
\end{tabular}




\section{Cuadro 8}

$\Delta\left(E D E V_{t}\right)=\gamma_{0}+\gamma_{1} \Delta\left(D E V_{t}\right)+\gamma_{2}\left(D E V_{t}-E D E V_{t-1}\right)+\gamma_{3}$ Intervencion $_{t}$

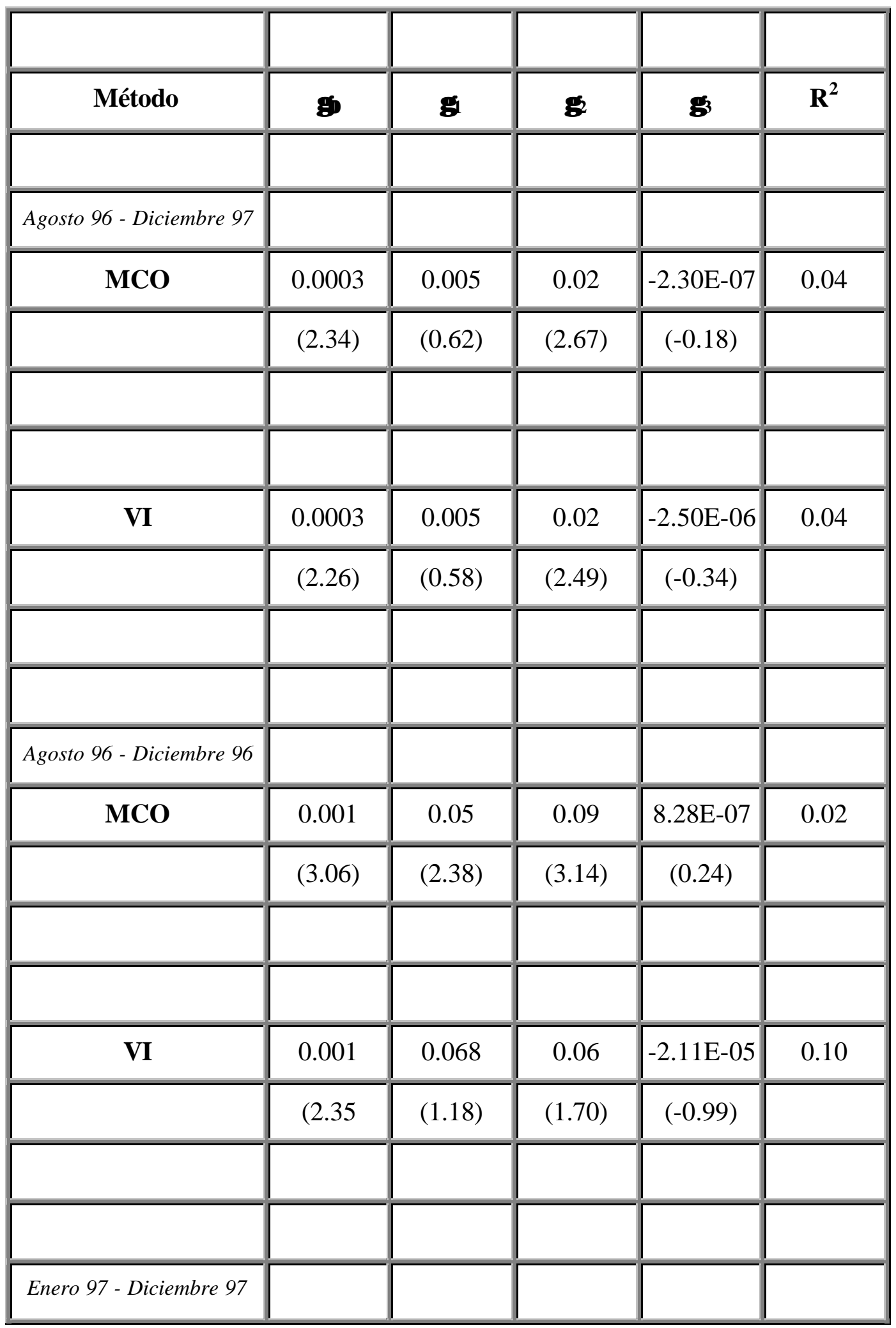




\begin{tabular}{|c|c|c|c|c|c|}
\hline MCO & 0.0002 & -0.01 & 0.02 & $-1.68 \mathrm{E}-07$ & 0.01 \\
\hline & $(1.59)$ & $(-1.56)$ & $(1.93)$ & $(-0.14)$ & \\
\hline & & & & & \\
\hline & & & & & \\
\hline & 0.0002 & -0.01 & 0.02 & $4.19 \mathrm{E}-06$ & -0.05 \\
\hline VI & $(1.37)$ & $(-1.56)$ & $(1.84)$ & $(0.51)$ & \\
\hline & & & & & \\
\hline
\end{tabular}

Los resultados de estas estimaciones, presentados en los cuadros 7 y 8 , demuestran que las intervenciones no han tenido ningún impacto sobre las expectativas de devaluación, ya que el coeficiente $\gamma_{3}$ nunca fue significativo. Estos resultados confirman el hecho de que las compras de dólares por parte del banco central mediante el mecanismo de opciones no han enviado ninguna señal al mercado sobre el curso futuro de la política monetaria o cambiaria.

Otras medidas que podrían mandar señales sobre la evolución futura de los determinantes fundamentales del tipo de cambio serían el anuncio de la implementación del programa de compra de activos internacionales, los anuncios del aumento en el monto de opciones a subastarse y el anuncio de la posibilidad de que en un mismo mes se incremente de manera contingente el monto de las opciones subastadas. A continuación, en el cuadro 9, se presentan los anuncios realizados con respecto al programa de acumulación de reservas y su impacto sobre el tipo de cambio y las tasas de interés el día del anuncio $\left(\Delta S_{t}\right.$ y $\left.\Delta i_{t}\right)$ y el día después del anuncio $\left(\Delta S_{t+1}\right.$ y $\left.\Delta i_{t+1}\right)$. 


\section{Cuadro 9}

\begin{tabular}{|c|c|c|c|c|c|}
\hline Fecha & Anuncio & & Efe & & \\
\hline & & $\Delta S_{\mathrm{t}}$ & $\Delta \mathrm{S}_{\mathrm{t}+1}$ & $\Delta \dot{\mathrm{i}}_{\mathrm{t}}$ & $\Delta \dot{\mathrm{i}}_{\mathrm{t}+1}$ \\
\hline 1/ago/96 & $\begin{array}{l}\text { Inicio del Programa } \\
130 \text { m.d.d. }\end{array}$ & $-0.24 \%$ & $-0.44 \%$ & -0.65 & -0.10 \\
\hline 27/ago/96 & Incremento a 200 m.d.d. & $0.19 \%$ & $0.31 \%$ & 0.05 & 0.01 \\
\hline 27/dic/96 & Incremento a 300 m.d.d. & $0.08 \%$ & $0.06 \%$ & 0.05 & 0.01 \\
\hline 19/feb/97 & $\begin{array}{l}\text { Se anuncia que en caso de } \\
\text { que se ejerza el } 80 \% \text { de las } \\
\text { opciones se convoca a otra } \\
\text { subasta por } 300 \text { m.d.d. } \\
\text { Se subastarán } 200 \text { m.d.d. en } \\
\text { caso de una depreciación de } \\
2 \% \text {. }\end{array}$ & $-0.15 \%$ & $-0.54 \%$ & -0.90 & 0.02 \\
\hline 30/jul/97 & Incremento a 500 m.d.d. & $-0.55 \%$ & $0.75 \%$ & 0.80 & -0.02 \\
\hline 29/sep/97 & Reducción a 400 m.d.d. & $-0.05 \%$ & $-0.58 \%$ & -0.65 & 0.02 \\
\hline 30/oct/97 & Reducción a 250 m.d.d. & $2.92 \%$ & $0.30 \%$ & 0.30 & -0.45 \\
\hline
\end{tabular}

En este cuadro no se detecta ningún efecto consistente de los anuncios de las ampliaciones del esquema de opciones sobre el tipo de cambio y las tasas de interés. 
Para evaluar el efecto de estos anuncios sobre el tipo de cambio y las tasas de interés, aislando el efecto de otras variables, se estimaron ecuaciones de determinación de las fluctuaciones cambiarias, de las expectativas de devaluación y de las tasas de interés que incorporan la posibilidad de que estas variables sean afectadas por los anuncios mencionados (Se excluyen los dos últimos anuncios ya que éstos deberían haber tenido el efecto contrario a los demás.). Las ecuaciones estimadas son las siguientes:

$$
\Delta S_{t}=B_{0}+B_{1} i_{t}^{*}+B_{2} i_{t-1}^{*}+B_{3} \text { Anuncio }(-1)+B_{4} i_{t-1}+B_{S^{i} t-2}+B_{6} S P_{t}+B_{7} S P_{t-1}
$$

$$
i_{t}=B_{0}+B_{1} i_{t}^{*}+B_{2} i_{t-1}^{*}+B_{3} \text { Anuncio }(-1)+B_{4} E D E V_{t}+B_{5} E D E V_{t-1}
$$

(11)

$$
+B_{8} S P_{t}+B_{7} S P_{t-1}+B_{8} i_{t-1}+B_{9} i_{t-2}+B_{10} i_{t-3}
$$

$$
\triangle E D E V_{t}=B_{0}+B_{1} \triangle D E V_{t}+B_{2}\left(D E V_{t}-E D E V_{t-1}\right)+B_{3} \text { Anuncio }(-1)
$$

$$
E D E V_{t}=B_{0}+B_{1} D E V_{t}+B_{2} E D E V_{t-1}+B_{3} \text { Anuncio }(-1)
$$

Estas ecuaciones intentan capturar el efecto de dichos anuncios en las fluctuaciones cambiarias, en las tasas de interés (ecuaciones (10) y (11)) y en las expectativas de depreciación de la moneda (ecuaciones (12) y (13)). Esta influencia se mide mediante el parámetro $B_{3}$ asociado a la variable Anuncio que toma el valor de 1 en aquellos días en los cuales se realizaron los anuncios referidos.

Los resultados de estas ecuaciones, que se presentan en el cuadro 10, confirman que estos anuncios no han tenido un efecto importante sobre el tipo de cambio, las expectativas de depreciación y las tasas de interés, ya que el coeficiente $B_{3}$ no es significativo en ningún caso. 


\section{Cuadro 10}

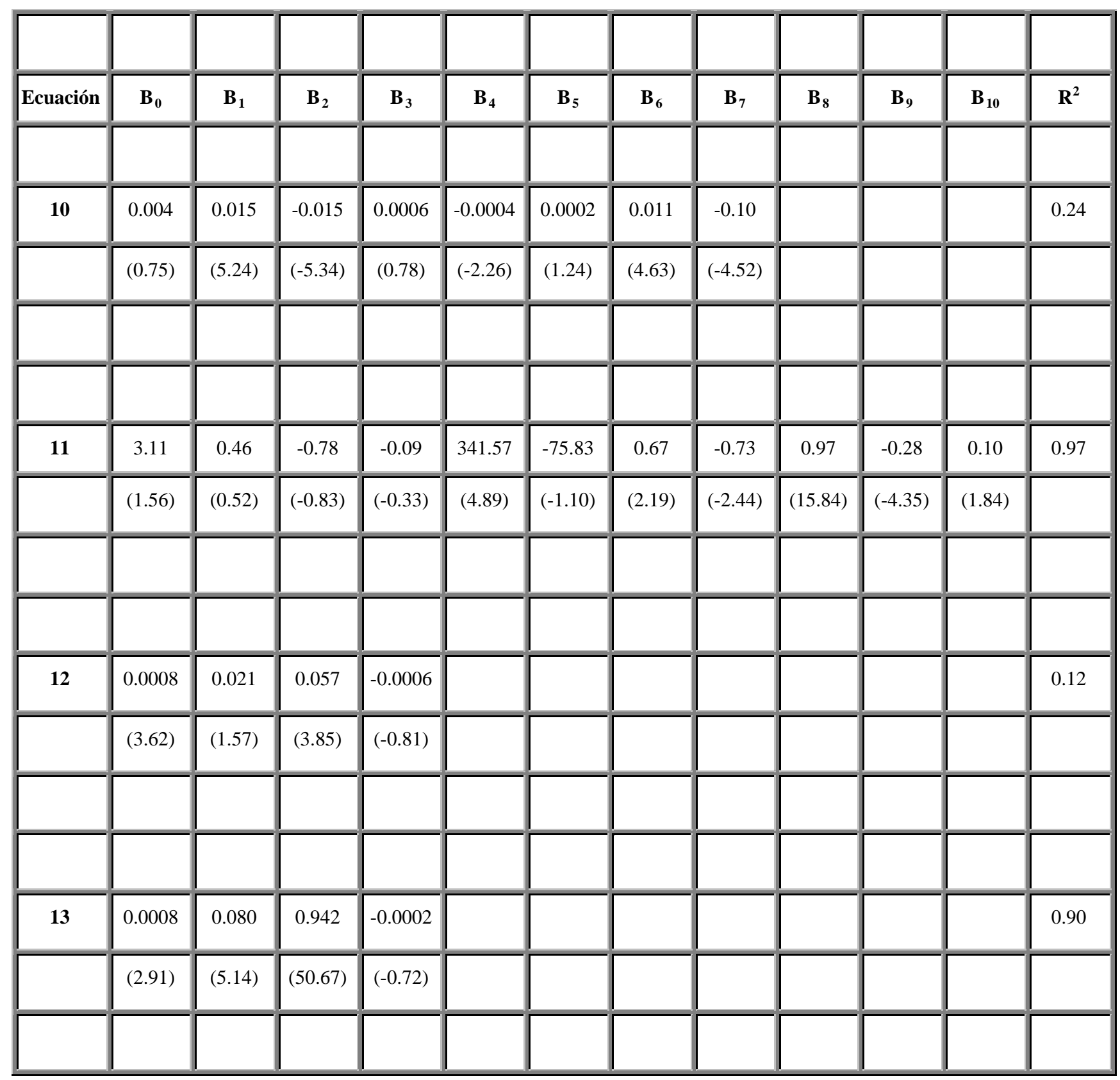




\section{III.- Conclusiones.}

De los ejercicios presentados se concluye que, hasta el momento, no se ha detectado un efecto importante de las intervenciones en el mercado cambiario y de su esterilización subsecuente en la determinación del tipo de cambio y las tasas de interés. Por otra parte, los ejercicios realizados en esta nota indican que durante los últimos meses de 1997, el efecto de las operaciones de esterilización sobre las tasas de interés fue estadísticamente significativo pero de una magnitud muy pequeña. Finalmente, se encontró cierta evidencia que durante los períodos en los cuales existe la posibilidad de que ocurran las intervenciones mediante opciones no ejercidas, las tasas de interés son ligeramente más elevadas que en los períodos en los que esta posibilidad no se presenta. Este resultado puede reflejar ciertas distorciones de caracter temporal que surjan en el mercado de dinero al realizar las operaciones de esterilización. 


\section{IV.- Bibliografía:}

Banco de México (1997) "Informe Anual 1996".

Branson, William (1979) "Exchange Rate Dynamics and Monetary Policy". En "Inflation and Employment in Open Economics". Editado por A. Lindbeck, North Holland.

Dornbusch, Rudiger (1983) "Exchange Risk and the Macroeconomics of Exchange Rate Determination". In Exchange Rates and Inflation, MIT Press.

Domínguez, Kathryn y Jeffrey Frankel (1993) "Does Foreign Exchange Intervention Work?". Institute for International Economics.

Edison, Hali (1993) "The Effectiveness of Central Bank Intervention: A Survey of the Post-1982 Literature". Special Papers on International Economics 18, Princeton University.

Frankel, Jeffrey (1982) "In Search of the Exchange Risk Premium: A Six Currency Test Assuming Mean Variance Optimization". Journal of International Money and Finance 1.

ITAM (1997) "La Política Monetaria en México". Suplemento de la Gaceta de Economía, Año 3, Número 5.

Mussa, Michael (1981) "The Role of Official Intervention". Group of Thirty Occasional Papers No. 6. New York: Group of Thirty.

Kaminsky, Graciela y Lewis, Karen (1993) "Does Foreign Exchange Intervention Signal Future Monetary Policy?". Finance and Economic Discussion Series No. 93-1. Washington, Federal Reserve Board. 
Lewis, Karen (1988) "Testing the Portfolio Balance Model: A Multi-Lateral Approach". Journal of International Economics 24.

Werner, Alejandro (1997) "Un Estudio Estadístico sobre el Comportamiento de la Cotización del Peso Mexicano Frenta al Dólar y de su Volatilidad". Banco de México, Documento de Trabajo No. 9701. 